
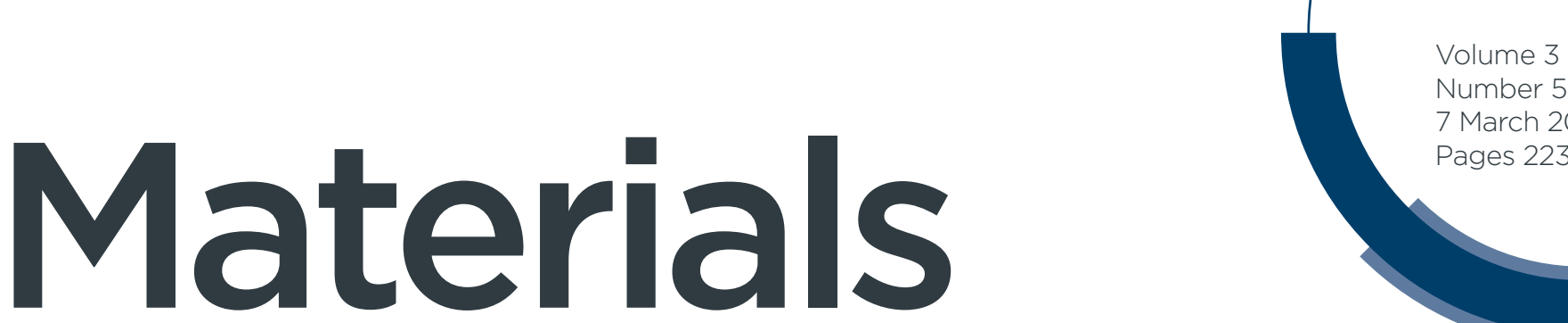

Number 5

7 March 2022

Pages 2237-2600

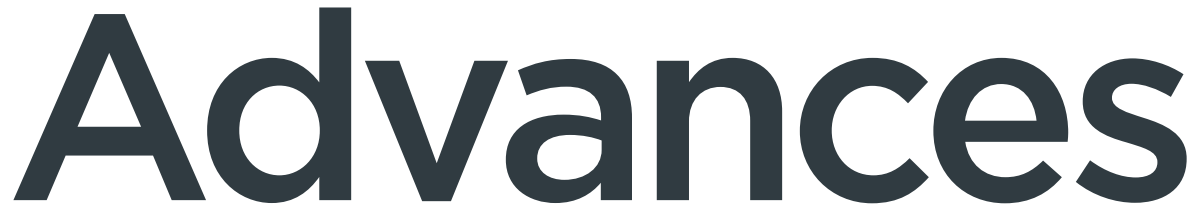

rsc.li/materials-advances

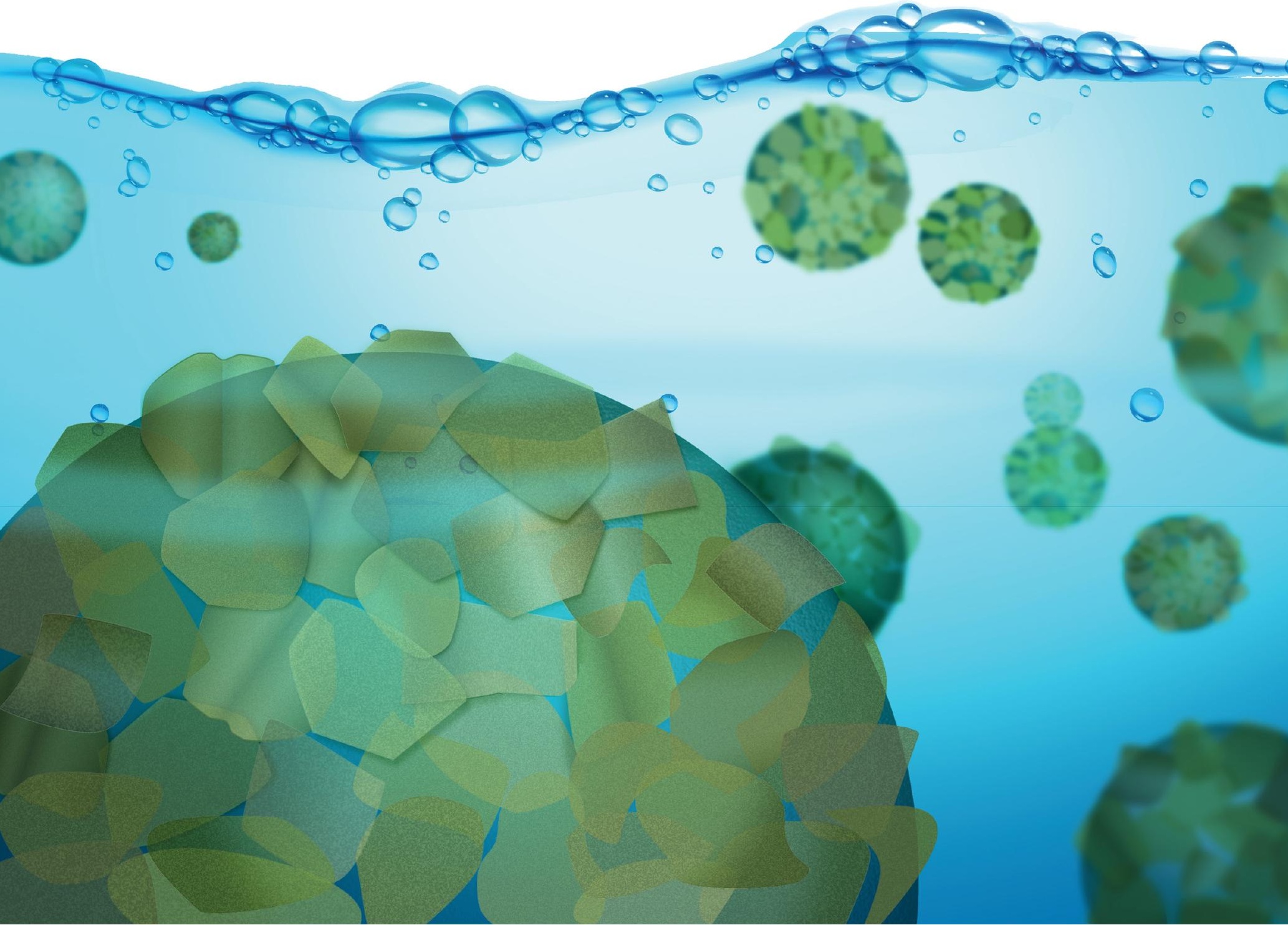

ISSN 2633-5409

ROYAL SOCIETY OF CHEMISTRY

\section{PAPER}

Emily Pentzer et al.

Polymer particles armored with cobalt oxide nanosheets for the catalytic degradation of bisphenol A 
Check for updates

Cite this: Mater. Adv., 2022, 3, 2354

Received 9th September 2021, Accepted 6th January 2022

DOI: $10.1039 / \mathrm{d} 1 \mathrm{ma} 00832 \mathrm{c}$

rsc.li/materials-advances

\title{
Polymer particles armored with cobalt oxide nanosheets for the catalytic degradation of bisphenol A $\dagger$
}

\author{
Maria Escamilla, (D) ${ }^{a}$ Kevin Pachuta, (D) ${ }^{b}$ Kuan Huang, (D ${ }^{c}$ Michael Klingseisen, ${ }^{d}$ \\ Huaixuan Cao, (D) e Huichun Zhang, (iD c Alp Sehirlioglu (D) ${ }^{b}$ and Emily Pentzer (D) *ad
}

\begin{abstract}
2D particle surfactants are attractive for the formation of highly stable emulsions and use as templates to prepare composite structures with performance properties dependent on the composition. Cobalt oxide nanosheets (CONs) are a relatively understudied class of $2 \mathrm{D}$ particle surfactants that can be produced by the chemical exfoliation of lithium cobalt oxide, a transition metal oxide known for excellent gas-sensing, catalytic, and electrochemical properties. Here, we report a simple method to access CONs stabilized oil-in-water Pickering emulsions and use these as templates to prepare particles with a core of polymer and shell of CONs. Salt-flocculated CONs produce emulsions with droplets of hydrophobic monomer (e.g., styrene) in water that are stable for at least 24 hours, and suspension free radical polymerization is used to produce $\mathrm{CON}$-armored particles. Characterization by $\mathrm{X}$-ray photoelectron spectroscopy (XPS), scanning electron microscopy (SEM), and thermal gravimetric analysis (TGA) confirmed the presence of CONs on the surface of the polymer core. We then demonstrated the CON-armored polymer particles can activate the oxidant peroxymonosulfate (PMS) for the degradation of bisphenol A (BPA). Freshly prepared and artificially aged CON-armored particles showed full degradation of BPA in less than an hour and no decrease in activity was observed after two uses. CONarmored particles combine high surface area of the nanosheets with the ease of recoverability of the particles.
\end{abstract}

\section{Introduction}

Emulsions find widespread industrial applications across pharmaceuticals, cosmetics, foodstuffs, and so on. Emulsions are classified as a mixture of two immiscible liquids, with droplets of one in a continuous phase of the other. Without the use of a surfactant, emulsions are typically kinetically unstable. Surfactants that reside at the interface of the two liquids can lower the interfacial tension and help form a stable phase separated system with a large amount of interfacial area. Oil-in-water

\footnotetext{
${ }^{a}$ Department of Chemistry, Texas A\&M University, College Station, TX, USA. E-mail: emilypentzer@tamu.edu, mxe180@case.edu

${ }^{b}$ Department of Materials Science and Engineering, Case Western Reserve University, Cleveland, OH, USA. E-mail: kgp22@case.edu, alp.sehirlioglu@case.edu

${ }^{c}$ Department of Civil and Environmental Engineering, Case Western Reserve University, Cleveland, OH, USA. E-mail: kxh515@case.edu, hjz13@case.edu

${ }^{d}$ Department of Materials Science and Engineering, Texas A\&M University College Station, TX, USA. E-mail: klingseisen79@tamu.edu

${ }^{e}$ Department of Chemical Engineering, Texas A\&M University, College Station, TX, USA. E-mail: chx744933979@tamu.edu

$\dagger$ Electronic supplementary information (ESI) available. See DOI: 10.1039/ d1ma00832c
}

emulsions are commonly stabilized by amphiphilic organic small molecules which possess a polar head and a non-polar tail (e.g., sodium dodecyl sulfate). In contrast, Pickering emulsions are those in which solid particles serve as a surfactant. Compared to small molecules, particle surfactants can lead to more stable emulsions and can have lower toxicity. ${ }^{1-3}$

Of particular interest as Pickering surfactants are 2D particles (e.g., nanosheets) which have high surface area and high aspect ratio; their organization parallel to the fluid-fluid interface leads to more surface area covered per particle compared to spherical counterparts, such as common $\mathrm{SiO}_{2}$ particles. ${ }^{4-6}$ Many research groups, including our own, have used graphene oxide (GO) nanosheets to stabilize oil-in-water emulsions, with more recent efforts towards modifying the surface of the nanosheets to enable them to stabilize other fluid-fluid combinations (e.g., ionic liquid-water, ionic liquid-oil, oil-oil, etc.). ${ }^{7-17}$ We have also recently demonstrated that transition metal carbide (MXene) and transition metal oxide (cobalt oxide) nanosheets can stabilize oil-in-water emulsions, if first flocculated by simple inorganic salt such as $\mathrm{NaCl} .{ }^{18,19}$ Compared to GO, these inorganic 2D surfactants are expected to not only provide kinetic stability to the emulsion, but to also impart 
favorable performance properties to any composite structures formed. Pickering emulsions stabilized by 2D particles have also gained increased popularity for use as templates to access composite structures, leveraging the confinement of reagents. For example, Janus particles can be prepared by using the interfacial assembly of nanosheets to effectively shield one face from a reagent. ${ }^{20,21}$ Alternatively, capsules are produced upon interfacial polymerization, or porous monoliths are prepared by polymerization of the discontinuous phase in high internal phase emulsions (HIPEs). ${ }^{22-26}$ If the discontinuous phase is polymerized, then armored particles are synthesized in which solid polymer particles are coated with nanosheets, which expose a large amount of nanosheet surface area. ${ }^{27-31}$

Cobalt oxide nanosheets (CONs) produced from the chemical exfoliation of, e.g., lithium cobalt oxide (LCO), and are a relatively understudied class of $2 \mathrm{D}$ particle surfactants but of great interest for potential catalytic, electrochemical, and superconductive properties. ${ }^{32-39}$ Limited studies have reported the use of cobalt oxide materials in emulsions. One example is the use of oil-in-water $(\mathrm{o} / \mathrm{w})$ Pickering emulsions to prepare composites of LCO nanoparticles and polyaniline as the positive electrode in a lithium battery; the discontinuous phase of the emulsion was aniline dissolved in toluene and LCO particles served as surfactant. ${ }^{40}$ Alternatively, Span-80 was used to stabilize aqueous droplets of lithium acetate and cobalt acetate in a continuous phase of kerosene for the synthesis of ultrafine LCO powder, with each droplet acting as a tiny reaction vessel. ${ }^{41}$ In a similar vein to the use of salt-flocculated LAPONITE $^{\circledR}$ clay nanosheets, ${ }^{42}$ our groups recently demonstrated that oil-in-water emulsions can be stabilized by a combination of CONs and GO nanosheets when flocculated with salt. ${ }^{19}$ In this previous work, the CONs were difficult to fully disperse in water (they were $0.8 \mathrm{~nm}$ thick and $\sim 1 \mu \mathrm{m}$ in diameter) and could not stabilize emulsions alone, as flocculation led to precipitation; this necessitated the use of GO nanosheets as co-surfactant. ${ }^{19}$ Recent advances in soft-chemical exfoliation of LCO gives access to CONs that are fully dispersible in water. ${ }^{43}$ Sonication aided in the exfoliation process and led to nanosheets with thickness of 2-4 $\mathrm{nm}$ and lateral widths of $>150 \mathrm{~nm}$. We hypothesized that these CONs could be used as the sole surfactant (e.g., without GO) to stabilize oil-in-water emulsions given they are easy to disperse in water, similar to GO. We further envisioned that suspension polymerization of the discontinuous phase in these emulsions would give access to polymer particles armored with CONs, that the properties of the exposed nanosheets could be leveraged for applicatoins, and that the particles could be easily isolated.

Herein we report the synthesis of CON-armored polymer particles and their use as an activator in an advanced oxidation process (Fig. 1), as relevant for organic contaminant removal from water. To prepare the $\mathrm{CON}$-armored particles, an aqueous dispersion of CONs was flocculated with inorganic salt, then an oil phase composed of radical initiator and olefin monomer was added and the system was agitated. Subsequent thermally induced free radical polymerization led to a solid polymer core coated (or "armored") with the nanosheets. The impacts of the salt identity on emulsion formation and stability are reported. We demonstrate the use of these CON-armored particles in the

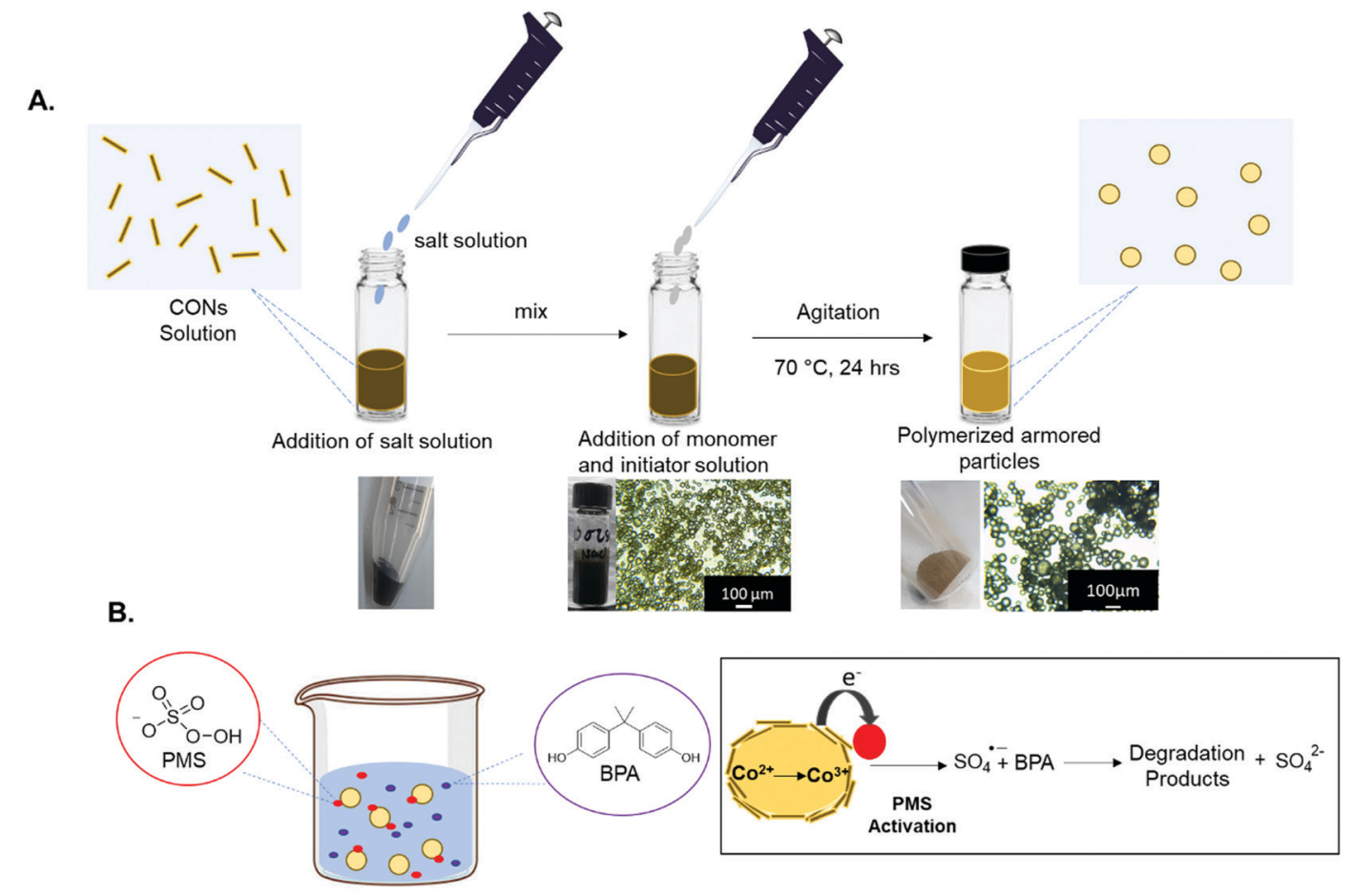

Fig. 1 (A) Overview of emulsion formation and preparation of CON-armored particles (photographs show synthesis of armored polybenzyl methacrylate particles), and (B) scheme for the degradation of BPA using CON-armored particles as catalyst to activate PMS. 
degradation of bisphenol A (BPA), a toxic compound widely used in plastics and epoxy resins. ${ }^{44}$ While the polymer particles without CONs did not show noticeable BPA degradation, the CON-armored particles led to rapid BPA degradation of $100 \%$ within 60 min. "Aging" of the armored particles by standing in water led to $100 \%$ removal of BPA in only $30 \mathrm{~min}$, which may be attributed to hydration of the nanosheets on the particle surface. Recyclability tests demonstrated that the CONs-containing catalyst could achieve stable BPA removal after the first run, making it promising for the catalytic degradation of BPA.

\section{Materials and methods}

\section{Materials}

Lithium cobalt oxide (LiCO, 98\%+) was purchased from MTI corporation, hydrochloric acid ( $\mathrm{HCl}, 37 \%[\mathrm{w} / \mathrm{w}]$, ACS reagent) was purchased from Fisher Scientific, tetramethylammonium hydroxide (TMAOH, 25\% [w/w], 99.9999\% electronic grade) was purchased from Alfa Aesar. $2 \mathrm{KHSO}_{5} \cdot \mathrm{KHSO}_{4} \cdot \mathrm{K}_{2} \mathrm{SO}_{4}$ (98\%), sodium hydroxide, sodium acetate, methanol, sulfuric acid, acetic acid, and bisphenol A (BPA) were purchased from Fisher Scientific. All reagents were used as received, without further purification. Benzyl methacrylate (96\%), styrene (99.9\%), $t$-butyl methacrylate (98\%), $n$-butyl methacrylate (99\%), and butyl acrylate $(>99 \%)$ were all purchased from Sigma-Aldrich and purified by passing through a basic alumina column prior to use. Azobisisobutyronitrile (AIBN, 98\%) was purchased from Sigma-Aldrich and purified by recrystallization in methanol and drying under reduced pressure.

\section{Instrumentation}

Emulsions were made using a combination of ultrasonication and agitation with a handheld emulsifier from BioSpec Products Inc. (model 985370). Optical microscopy images were obtained by placing a drop of the emulsion on a glass slide and using an amscope microscope. X-Ray photoelectron spectroscopy (XPS) was performed using an Omicron X-ray photoelectron spectrometer employing a Mg-sourced X-ray beam at $15 \mathrm{kV}$ with aperture 3. Scanning electron microscopy (SEM) and energy dispersive X-ray spectroscopy (EDS) was conducted on a FEI Quanta 600 field-emission scanning electron microscope with the acceleration voltage of $5 \mathrm{kV}$ and $20 \mathrm{kV}$ for microscopy imaging and energy-dispersive spectroscopy. UV-vis spectra were collected using an integrating sphere on a Cary $6000 \mathrm{i}$ UV-Vis NIR Spectrophotometer with absorbance collected in transmission mode from $1075 \mathrm{~nm}$ to $260 \mathrm{~nm}$ with background subtraction. Atomic force microscopy (AFM) was performed on a sample drop cast onto freshly cleaved mica and imaging with a Park Nanosystems NX10 in tapping mode. BPA concentration studies were carried out by high-performance liquid chromatography (HPLC, Agilent 1260 Infinity II) equipped with a C-18 column (Zorbax XDB-C18; $4.6 \times 250 \mathrm{~mm}^{2}, 5 \mu \mathrm{m}$ ) and a diode array detector.

\section{Preparation of CONs}

A suspension of cobalt oxide nanosheets (CONs) dispersed in deionized (DI) water was obtained using the soft-chemical exfoliation method described in detail elsewhere. ${ }^{43}$ Briefly, as received lithium cobalt oxide (LCO) powder was treated with $3 \mathrm{M}$ hydrochloric acid ( $\mathrm{HCl})$ for 1 day using constant stirring. The acid-treated powder was washed with copious amounts of DI water to remove residual acid or salt and allowed to air dry. This acid-treated powder was immersed in $0.044 \mathrm{M}$ aqueous tetramethylammonium hydroxide (TMAOH) for 1 day using constant stirring, resulting in formation of a dark slurry. Upon centrifugation (3260 ref for 15 minutes), a pellet composed of larger semi-exfoliated TMAOH-treated particles was isolated and the supernatant was discarded. This TMAOH-treated powder was washed with multiple aliquots of DI water until the wash supernatant solution was $\mathrm{pH}$ neutral, as determined by Litmus test ( $\sim 50 \mathrm{~mL}$ total volume). Additional DI water was added to the pellet and the system subjected to bath sonication for one hour, producing a highly concentrated suspension of CONs in DI water, as evident by the dark color. This suspension was centrifuged (3260 rcf for 5 minutes) to isolate any remaining non-exfoliated particles to give a supernatant which contained CONs as a pH neutral aqueous suspension, which were used for the experiments described below. Treatment with $\mathrm{HCl}$ and TMAOH were performed with a concentration of $10 \mathrm{mg}$ of powder per $\mathrm{mL}$ of solution. Finally, the concentration of the CONs suspension was determined through mass analysis of the particles that remained from a known volume of evaporated CONs suspension.

\section{Flocculation of CONs, emulsion preparation, and CON- armored particle preparation}

The CONs/salt solutions were made with an aqueous CONs concentration of $1.2 \mathrm{mg} \mathrm{mL} \mathrm{mL}^{-1}$ and salt concentrations of $0.0025 \mathrm{M}, 0.05 \mathrm{M}, 0.05 \mathrm{M}$, and $0.1 \mathrm{M}$. Upon addition of aqueous solutions of salt, the dispersed nanosheets aggregated to form small visible flocculates. The preparation of the emulsions was conducted using a monomer as the oil phase. For example, a solution of AIBN (5 wt $\%$ relevant to monomer) in styrene $(0.4 \mathrm{~mL})$ was added to a $2 \mathrm{~mL}$ solution of the $\mathrm{NaCl} / \mathrm{CONs}$ suspension. The mixture was vortexed for approximately 10 seconds and then subjected to bath sonication for 2 minutes, followed by agitation with a handheld emulsifier for a total of 1 minute (three 20 second intervals with a 5 second pause in between) at maximum power. Armored particles were synthesized by placing the vial containing the emulsion on an aluminum block heated to $70{ }^{\circ} \mathrm{C}$ and leaving unagitated for 24 hours. The armored particles were isolated by gravity filtration, then washed with deionized water (thrice) then methanol (thrice) and dried under reduced pressure at $22{ }^{\circ} \mathrm{C}$ for 24 hours.

\section{Preparation of non-armored polymer particles}

As control samples, polymer particles were prepared by suspension polymerization using emulsions stabilized by a small molecule surfactant. Briefly, the oil phase $(2 \mathrm{~mL})$ consisted of 
the styrene and AIBN (5 wt\% relevant to monomer) and the aqueous continuous phase was comprised of deionized water $(10 \mathrm{~mL})$ and $2.4 \mathrm{mg}$ sodium lauryl sulfate $(8.5 \mathrm{mM})$. The oil phase was added to the aqueous solution and agitated using a handheld emulsifier (three 20 second intervals with a 5 second pause in between). The emulsion was placed on a hot plate at $70{ }^{\circ} \mathrm{C}$ for 24 hours. The polymer particles were isolated by gravity filtration, washed (thrice with both deionized water and hexane), and dried under reduced pressure for 24 hours.

\section{Catalytic oxidation of BPA using PMS as the oxidant and CON- armored particles as catalyst}

The oxidation of BPA using PMS was activated using the CONarmored particles; here, the armored particles serve as the catalyst and PMS as the oxidant. This reaction was performed in a $50 \mathrm{~mL}$ beaker at room temperature with a magnetic stirring at $400 \mathrm{rpm}$ using $30 \mathrm{~mL}$ of reaction mixture containing $0.04 \mathrm{~g} \mathrm{~L} \mathrm{~L}^{-1}$ catalyst, $500 \mu \mathrm{M}$ PMS, and $5 \mu \mathrm{M}$ BPA at $\mathrm{pH} 5$ controlled with $0.02 \mathrm{M}$ acetate buffer ( $\mathrm{pH}$ changes throughout the reaction were less than 0.2 units). Note that the "catalyst" refers to the particles as a whole instead of the functional fraction (i.e., CONs). The change in concentration of BPA was monitored by withdrawing small aliquots at predetermined time intervals from the reaction mixture into $3 \mathrm{~mL}$ syringe then passting it through a $0.2 \mu \mathrm{m}$ filter. Methanol $(0.5 \mathrm{~mL})$ was pre-loaded into the syringe to quench the radicals produced. ${ }^{44}$ The aliquot was analyzed by high-performance liquid chromatography, with the detection wavelength set at $279 \mathrm{~nm}$ (BPA) and the mobile phase contained $0.1 \%$ acetic acid and methanol at a ratio of $57: 43(\mathrm{v} / \mathrm{v})$; BPA retention time was $5.74 \mathrm{~min}$.

For reactions involving two reactants as shown in eqn (1), second order kinetic model is generally used to describe the reactions and calculate the involved rate constant $k$ (eqn (2)). The curly brackets \{\} represent the activity of corresponding species, which is approximately equal to the concentration shown as square brackets [] under low ionic strength conditions. However, it is usually hard to calculate $k$ as the concentrations of the two reactants change simultaneously. Therefore, to simplify this process, a common practice is to increase the oxidant concentration to a much higher level so that it can be regarded as a constant, resulting in a pseudo-first order model (eqn (3)). Therefore, the pseudo-first order rate constant $k^{\prime}$ can be easily obtained as the slope of the plot $\ln \left([\mathrm{B}]_{t} /[\mathrm{B}]_{0}\right) v s$. time $t$.

$$
\mathrm{A}(\text { oxidant })+\mathrm{B}(\text { pollutant }) \stackrel{k}{\rightarrow} \mathrm{C}
$$

$\mathrm{R}=k\{\mathrm{~A}\}\{\mathrm{B}\} \approx k[\mathrm{~A}][\mathrm{B}]$ (under low ionic strength condition)

$$
\mathrm{R}=k^{\prime}[\mathrm{B}] \frac{\Delta B}{\Delta t}\left(k^{\prime}=k[\mathrm{~A}]\right)
$$

To evaluate the reusability of the catalysts, two different CON-armored particles (polystyrene and polybenzyl methacrylate) were coated onto graphite sheets, and used for the catalytic removal of BPA. This was done to facilitate recovery of the particles. To prepare these sheets, $120 \mathrm{mg}$ of catalyst and $150 \mu \mathrm{L}$ of the Nafion solution (15\% in alcohol, Ion Power Inc.) were added to $10 \mathrm{~mL}$ of isopropanol. The Nafion solution was the polymer binder to ensure the stable coating of the armored particles onto the graphite sheet. The mixture was ultrasonicated for $1 \mathrm{~h}$, then $100 \mu \mathrm{L}$ of the resulting solution was added onto one side of a graphite sheet $(2.5 \mathrm{~cm} \times 2.5 \mathrm{~cm} \times 0.5 \mathrm{~mm})$. After 20 min of drying at $55{ }^{\circ} \mathrm{C}$, another $100 \mu \mathrm{L}$ of the solution was added onto the same side of the sheet and then dried. This process was repeated on the other side of the sheet to obtain an evenly coated graphite sheet with $4.8 \mathrm{mg}$ of the CON-armored particles. $^{45,46}$

\section{Results and discussion}

\section{Preparation and characterization of CONs}

Cobalt oxide nanosheets (CONs) were prepared as previously reported by a two-step procedure (Fig. S1, ESI $\dagger$ ): (1) as-received lithium cobalt oxide (LCO) was treated with $\mathrm{HCl}$; then (2) the acid-treated LCO was treated with an aqueous solution of tetramethylammonium hydroxide (TMAOH) to produce TMAOH-treated LCO. ${ }^{35,43,47-49}$ The traditional exfoliation method used to produce CONs from LCO yields only a low concentration of nanosheets in highly caustic solution (i.e., excess of TMAOH). The modified exfoliation method used here utilized semi-exfoliated TMAOH-treated LCO, rather than the initially exfoliated nanosheets to produce highly concentrated aqueous dispersions of CONs at neutral $\mathrm{pH}^{43}$ by immersing semi-exfoliated TMAOH-treated LCO particles in DI water and subjecting to bath sonication. After centrifugation, the supernatant was an aqueous dispersion of CONs. The UV-vis spectrum of the aqueous CONs suspension shows three absorbance peaks characteristic of the 2D nanosheets (Fig. S2A, ESI $\dagger$ ). This dispersion was drop-cast onto freshly cleaved mica and characterized by atomic force microscopy (AFM), revealing 2D particles with thickness (i.e., heights) of $\sim 4 \mathrm{~nm}$ and lateral widths of $\sim 150 \mathrm{~nm}$ (Fig. S2B, ESI $\dagger$ ).

\section{CONs stabilized emulsions}

We tested the CONs as the sole particle surfactants for oil-inwater emulsions, by using $1.2 \mathrm{mg} \mathrm{mL} \mathrm{mL}^{-1}$ of aqueous CONs solution and styrene as the oil phase in a 1:5 oil: water ratio. Emulsification by shear mixing led to formation of large, semispherical droplets which, upon being left unagitated for 24 hours, became less uniform in size and were accompanied by large dark masses (Fig. S3A, ESI $\dagger$ ). We hypothesize these dark structures are aggregated nanosheets. Based on our previous work with MXenes and GO/CONs as particle surfactants, we then evaluated the use of inorganic salt to flocculate CONs and facilitate their use as surfactants. Addition of aqueous solutions of $\mathrm{NaCl}(0.0025 \mathrm{M}, 0.005 \mathrm{M}, 0.025 \mathrm{M}, 0.05 \mathrm{M}$, $0.1 \mathrm{M}$ ) to the aqueous CONs dispersion resulted in the obvious flocculation of the nanosheets, but did not lead to their precipitation (Fig. S4A, ESI $\dagger$ ). After flocculation, styrene was added, and the system agitated by shear mixing. Fig. 2 displays 


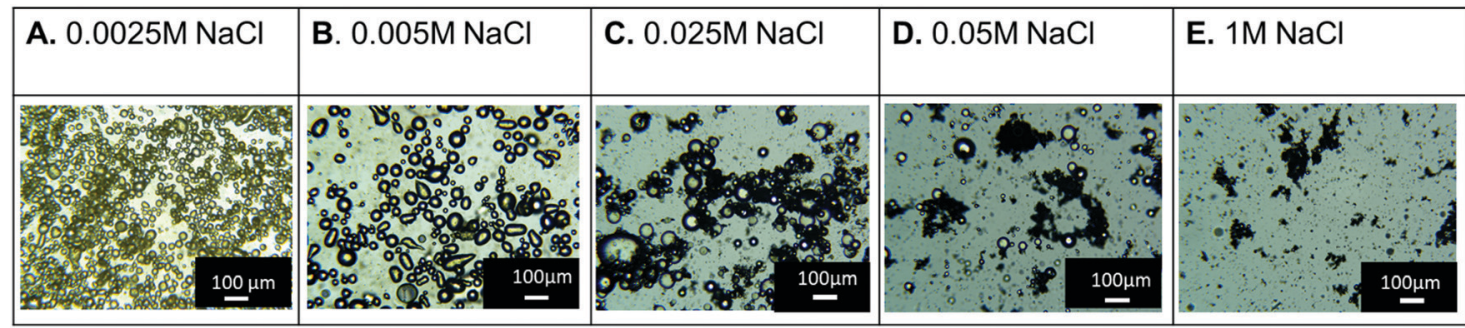

Fig. 2 Styrene-in-water emulsions stabilized by CONs flocculated with (A) $0.0025 \mathrm{M} \mathrm{NaCl}$, (B) $0.005 \mathrm{M} \mathrm{NaCl},(C) 0.025 \mathrm{M} \mathrm{NaCl}$, (D) $0.05 \mathrm{M} \mathrm{NaCl}$, and (E) $0.1 \mathrm{M} \mathrm{NaCl}$. An aqueous dispersion of $1.2 \mathrm{mg} \mathrm{mL}^{-1}$ of CONs was used and a 1:5 oil : water ratio.

the optical microscopy images of the resulting emulsions and the impact of salt concentration. From these images, the three highest salt concentrations $(0.025 \mathrm{M}, 0.05 \mathrm{M}, 1 \mathrm{M})$ lead to larger aggregates of CONs and lack of well-defined droplets (Fig. 2C-E). However, at the lower salt concentrations the emulsion droplets were spherical and fewer dark, ill-defined aggregates were observed; this supports that the nanosheets were associated with the fluid-fluid interface. To determine stability, the emulsions were left unagitated for 24 hours then optical microscopy images were again taken; droplets of similar size to those immediately after emulsion formation were observed at lower salt concentrations $(0.0025 \mathrm{M}$ or $0.005 \mathrm{M}$ $\mathrm{NaCl}$, Fig. S4C and S5, ESI $\dagger$ ). From these studies, the best conditions for emulsion formation were identified as flocculating a $1.2 \mathrm{mg} \mathrm{mL}^{-1}$ aqueous CONs solution using 0.0025 or $0.005 \mathrm{M}$ of $\mathrm{NaCl}$.

Other common inorganic salts were then evaluated as flocculating agents for CONs, specifically $\mathrm{LiCl}, \mathrm{CsCl}$, and $\mathrm{MgCl}_{2}$ (Fig. S6, ESI $\dagger$ ). The optical microscopy images of the resulting emulsions revealed that, of these additional salts, only LiCl led to the formation of spherical droplets, whereas $\mathrm{MgCl}_{2}$ and CsCl showed large aggregates of nanosheets in the continuous phase. The formation and stability of emulsions prepared with LiCl-flocculated CONs was evaluated after 24 hours, using salt concentrations of $0.0025 \mathrm{M}, 0.005 \mathrm{M}, 0.025 \mathrm{M}, 0.05 \mathrm{M}$, and $0.1 \mathrm{M}$ (Fig. S7, ESI $\dagger$ ). Similar to $\mathrm{NaCl}$, optical microscopy images showed that lower salt concentrations $(0.005 \mathrm{M})$ produced the most stable emulsions. These results are in line with our recent work using salt flocculated MXene nanosheets to stabilize oil-in-water emulsions and may indicate that different cation radius or "hardness" dictates the ability to use inorganic nanosheets to stabilize Pickering emulsions. ${ }^{18}$ Notably, the difference in ionic strength of $0.005 \mathrm{M}$ solutions for monovalent and divalent cations may also play a role (see Fig. S6, ESI $\dagger$ ).

\section{CON-armored particle formation and characterization}

A series of polymer particles armored with CONs were synthesized, templated by the Pickering emulsions described above. Both $\mathrm{LiCl}$ and $\mathrm{NaCl}$ were used as flocculating agents. First, an oil-in-water emulsion was formed $\left(1.2 \mathrm{mg} \mathrm{mL}^{-1}\right.$ of an aqueous CONs solution flocculated with $0.0025 \mathrm{M}$ and $0.005 \mathrm{M} \mathrm{LiCl}$ or $\mathrm{NaCl}$ in a $1: 5$ oil and water ratio). The oil phase was composed of azobisisobutyronitrile (AIBN) dissolved in styrene (5 wt\% to monomer) and the prepared emulsions were heated to $70{ }^{\circ} \mathrm{C}$ for $24 \mathrm{~h}$. After cooling to room temperature, the armored particles were isolated by gravity filtration and washed with water, then methanol, and dried under reduced pressure. Out of the four conditions used $(0.0025 \mathrm{M}$ and $0.005 \mathrm{M}$ with $\mathrm{LiCl}$ or $\mathrm{NaCl})$, optical microscopy images of the armored particles support that flocculation with $0.0025 \mathrm{M} \mathrm{NaCl}$ produced the "best" particles, based on particle uniformity, shape, and ability to be redispersed in water (Fig. S8, ESI $\dagger$ ).

We then leveraged these conditions to prepare armored particles with different polymer cores, evaluating monomers of different polarity: benzyl methacrylate, $n$-butyl methacrylate, $t$-butyl methacrylate, and $n$-butyl acrylate (Fig. 3). The emulsions were all prepared following the same procedure as described above and yielded well-defined emulsion droplets. After polymerization, all systems formed well-defined and dispersed spherical particles, as determined by optical microscopy (Fig. 3). However, after isolation (filtration and washing), only the armored particles synthesized from benzyl methacrylate could be isolated as powder, similar to the polystyrene armored particles. Poly( $n$-butyl methacrylate) particles, once dried, gave aggregates that were sticky, while poly(t-butyl methacrylate) and poly(n-butyl acrylate) samples were hard monolithic solids. The lack of isolated individual particles for these monomers could be due to differences in extent of polymerization, $T_{\mathrm{g}}$ of the polymer core, or different affinity of CONs for the more polar monomers.

The morphology, composition, and thermal properties of the CON-armored polymer particles were characterized by thermogravimetric analysis (TGA), energy dispersive X-ray spectroscopy (EDS) scanning electron microscopy (SEM), and X-ray photoelectron spectroscopy (XPS). Using TGA, the thermal degradation of the polymers alone and the CON-armored particles were compared (Fig. S9, ESI $\dagger$ ); briefly, no significant difference in the thermal degradation was observed, which may be owing to the low loading of CONs calculated to be $\sim 0.66 \mathrm{wt} \%$ based on feedstock, and supported by energy dispersive spectroscopy (EDS, Fig. S10, ESI $\dagger$ ). The SEM images of the armored particles show that they are spherical with a rough surface supporting the presence of CONs on the surface (Fig. 4A). In contrast, polystyrene particles prepared using the small molecule surfactant sodium lauryl sulfate have smooth surfaces (Fig. S11, ESI $\dagger$ ). The XPS survey spectrum of the CONarmored particles showed the presence of C (73.5\%), O (22.3\%), 


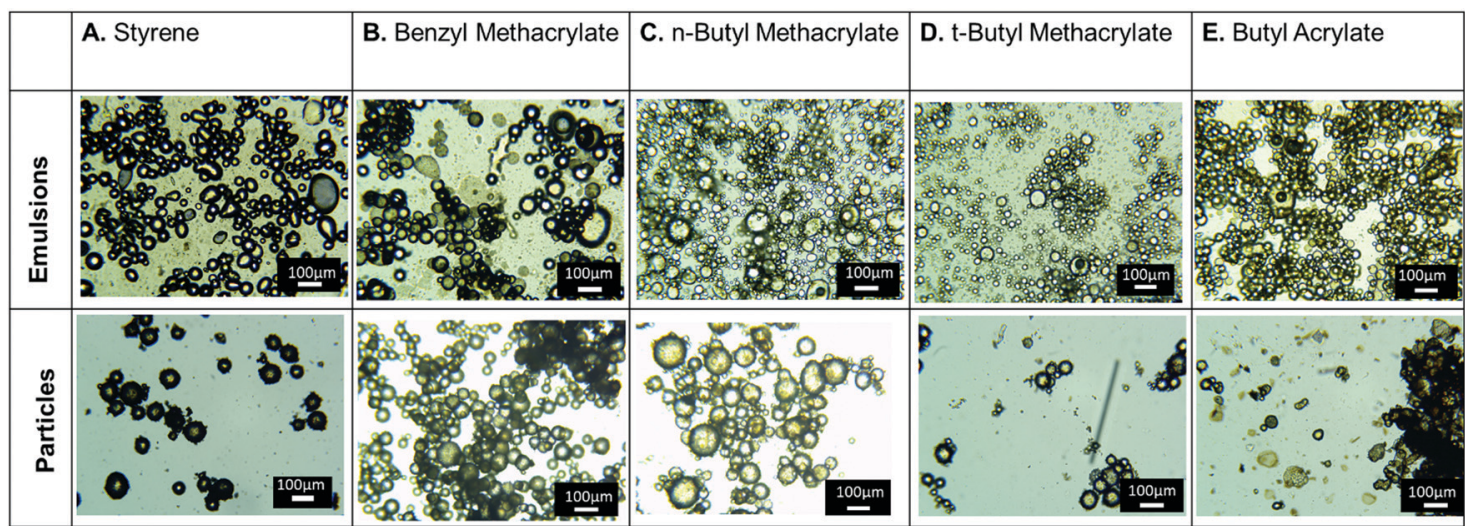

Fig. 3 Optical microscopy images of emulsions (top) stabilized by salt-flocculated CONs and their respective CON-armored particles (bottom) prepared using: (A) styrene, (B) benzyl methacrylate, (C) $n$-butyl methacrylate, (D) t-butyl methacrylate, and (E) $n$-butyl acrylate. $1.2 \mathrm{mg} \mathrm{mL}^{-1}$ of flocculated CONs and $0.0025 \mathrm{M} \mathrm{NaCl}$ were used.

A.
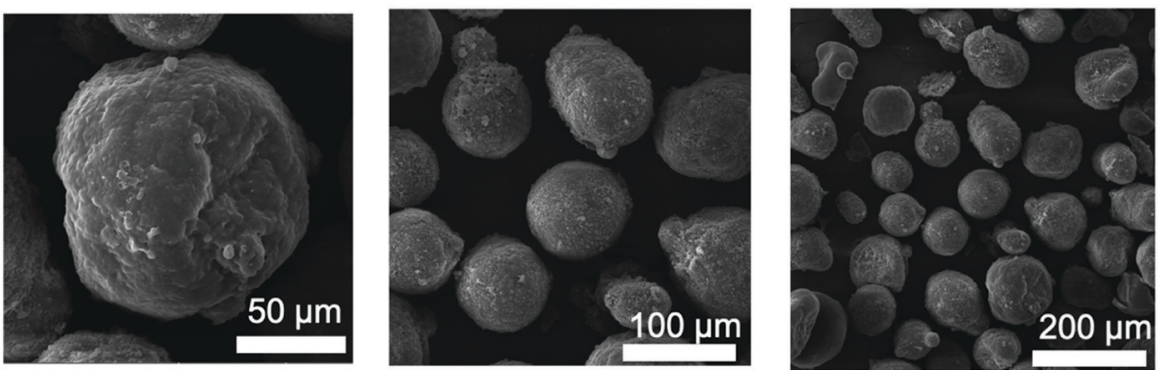

B.

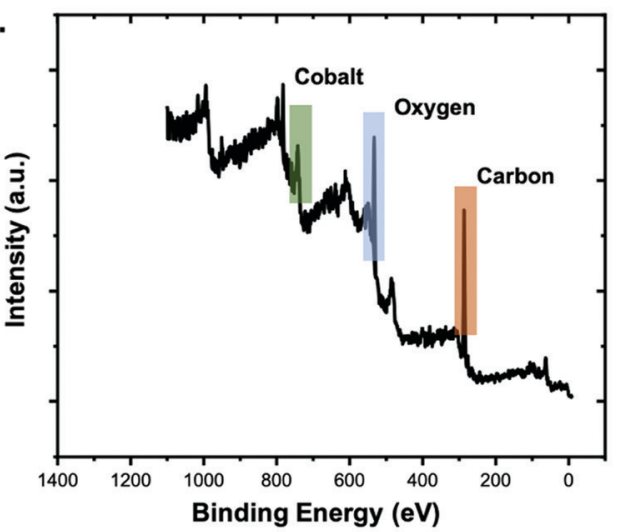

C.

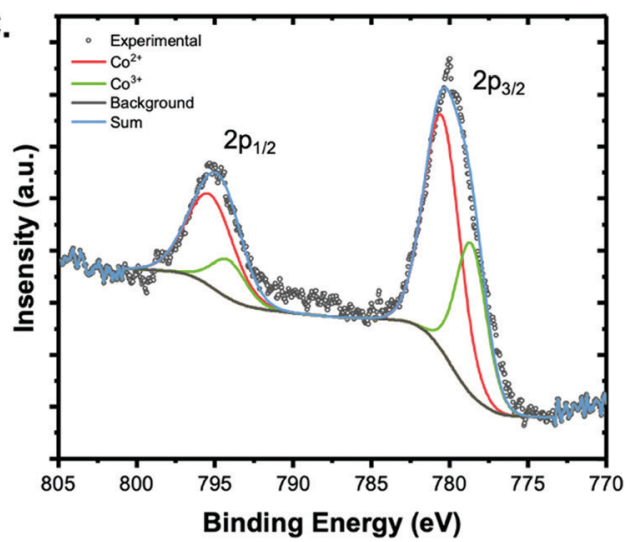

Fig. 4 Characterization of CON-armored polystyrene particles: (A) SEM images at different magnification, (B) XPS survey spectrum, and (C) high resolution Co $2 \mathrm{p}$ peak of the XPS spectrum.

and Co $(4.12 \%)$, indicating the presence of both polymer and CONs (Fig. 4B). Fig. 4C is the high-resolution spectrum of the Co 2p peak; the two peaks indicate the mixed oxidation state of cobalt $\left(68 \% \mathrm{Co}^{2+}\right.$ and $\left.32 \% \mathrm{Co}^{3+}\right)$, as expected for chemically exfoliated CONs.

\section{Catalytic oxidation of BPA using CON-armored particles}

To establish the application of the CON-armored particles for environmental remediation, we used them to activate peroxymonosulfate (PMS), an oxidant, for the degradation of bisphenol A (BPA) in water. We envisioned that the CON-armored particle would provide a relatively high surface area of the nanosheets at minimal nanosheet composition $(0.66 \mathrm{wt} \%$ of particle), and that the particles could be more easily recovered than dispersed nanosheets which require centrifugation. PMS was chosen as an oxidant due to its high stability, high efficiency, low cost, and high solubility in water. Although PMS itself is not very reactive towards organic pollutants (as for many oxidants such as $\mathrm{H}_{2} \mathrm{O}_{2}$ and peroxydisulfate), it can be activated by a catalyst through a series of redox reactions to generate reactive oxygen species (ROS). ${ }^{50}$ The ideal catalyst can maintain its activity for a relatively long time without 
attenuation, which can be achieved if it is able to periodically return to its initial state during the catalytic reactions. Such a process usually creates reaction cycles that continuously activate PMS without consuming the catalyst, as shown in eqn (4)(8). For PMS, transition metal oxides have been reported to have such properties. ${ }^{51,52}$

An overview of the reactions between CONs, PMS, and BPA are shown in eqn (4)-(8). $\mathrm{HSO}_{5}{ }^{-}$first reacts with $\mathrm{Co}^{2+}$ forming a $\mathrm{SO}_{4}{ }^{\bullet-}$ and $\mathrm{Co}^{3+}$ (eqn (4)). The formed $\mathrm{Co}^{3+}$ then reacts with another $\mathrm{HSO}_{5}{ }^{-}$to generate $\mathrm{SO}_{5}{ }^{--}$and $\mathrm{Co}^{2+}$ (eqn (5)), thus returning the cobalt back to its initial state $\left(\mathrm{Co}^{2+}\right)$. Two molecules of the formed $\mathrm{SO}_{5}{ }^{--}$can react with each other to form $\mathrm{SO}_{4}{ }^{\bullet-}$ and $\mathrm{O}_{2}$ (eqn (6)). Although the $\mathrm{SO}_{4}{ }^{\bullet-}$ can also react with $\mathrm{OH}^{-}$to form ${ }^{\bullet} \mathrm{OH}$ at high $\mathrm{pH}$ (eqn (7)), this pathway is not expected to be significant based on solution $\mathrm{pH}(\mathrm{pH}=5)$. As a result, we expect $\mathrm{SO}_{4}{ }^{--}$to be the major ROS responsible for BPA oxidation (eqn (8)).

$$
\begin{gathered}
\mathrm{HSO}_{5}{ }^{-}+\mathrm{Co}^{2+} \rightarrow \mathrm{SO}_{4}^{\bullet-}+\mathrm{Co}^{3+}+\mathrm{OH}^{-} \\
\mathrm{HSO}_{5}^{-}+\mathrm{Co}^{3+} \rightarrow \mathrm{SO}_{5}^{\bullet-}+\mathrm{Co}^{2+}+\mathrm{H}^{+} \\
2 \mathrm{SO}_{5}^{\bullet-} \rightarrow 2 \mathrm{SO}_{4}^{\bullet-}+\mathrm{O}_{2} \\
\mathrm{SO}_{4}^{\bullet-}+\mathrm{OH}^{-} \rightarrow{ }^{\bullet} \mathrm{OH}+\mathrm{SO}_{4}{ }^{2-} \\
\mathrm{SO}_{4}^{\bullet-}+\mathrm{BPA} \rightarrow \text { products }+\mathrm{SO}_{4}{ }^{2-}
\end{gathered}
$$

The catalytic efficiencies of CON-armored polystyrene particles and CON-armored polybenzyl methacrylate particles for PMS activation were evaluated and compared to polymer particles not armored with CONs (i.e., prepared with an organic surfactant). As expected, non-armored polystyrene and polybenzyl methacrylate particles did not lead to detected degradation of BPA, either on their own or when coupled with PMS (Fig. S12, ESI $\dagger$ ). In contrast, the CON-armored polybenzyl methacrylate particles showed rapid BPA degradation in the presence of PMS (Fig. S12, ESI $\dagger$ ). As a comparison, no change in BPA concentration was observed under the experiments of peroxydisulfate/catalyst and UV/catalyst, which are two other common catalytic AOPs (data not shown). This indicates the prepared catalysts are mainly active toward PMS.
The CON-armored polystyrene particles showed full degradation of BPA within 60 min (Fig. 5A), faster than that of CONarmored polybenzyl methacrylate particles (Fig. S12B, ESI $\dagger$ ). This difference in degradation rate may be attributed to the available surface area of the nanosheets based on better miscibility with polybenzyl methacrylate or slightly smaller size of these particles (Fig. S13, ESI $\dagger$ ). The kinetics of BPA degradation were found to follow the pseudo-first order model with a rate constant $k$ of $0.065 \mathrm{~min}^{-1}$ and an $R^{2}$ of 0.998 (Fig. 5A, inset). To test the stability of these composite structures, the CONarmored polystyrene particles were left in a solution of acetate buffer for one month and then their catalytic efficiency was tested; after this "aging", full degradation of BPA occurred within 30 minutes (Fig. 5B) - half the time it took for freshly prepared samples. The pseudo-first order rate constant reached $0.137 \mathrm{~min}^{-1}$ (Fig. 5B, inset), an increase of $111 \%$ from the original value. Although surprising, this difference in activity may be attributed to the degree of hydration of the CONs on the polymer particles and may indicate a route to better performance.

The CON-armored polystyrene and polybenzyl methacrylate particle catalysts were also fixated onto graphite sheets to determine their efficacy as a monolithic sample over multiple cycles. In the activation of PMS, many catalysts show decreasing activity upon repeated use. For example, Ahn et al. used surfaceloaded noble metal nanoparticles as catalyst $\left(\mathrm{Pd} @ \mathrm{TiO}_{2}\right)^{53}$ and observed removal efficiency decreased quickly from $100 \%$ to $92 \%, 81 \%, 75 \%$, and $52 \%$, during five repeated uses. Alternatively, hexagonally-ordered mesoporous carbon as catalyst led to decrease in removal efficiency from $100 \%$ to $98.8 \%, 87 \%$, and $72 \%$, over the four cycle run, ${ }^{54}$ whereas $\mathrm{CoFe}_{2} \mathrm{O}_{4}$ as catalyst led to removal decrease from $>99 \%$ to $65 \%$ over a five cycle run. ${ }^{55}$ As shown in Fig. 5C and Fig. S14 (ESI $\dagger$ ), both the CONarmored particles showed lower BPA removal efficiencies for the second cycle than the first, indicating decreased performance which may be due to adsorption of BPA or degradation byproducts $^{54,56}$ or possibly delamination of loosely bound CONs. However, the third and fourth cycles did not show further decrease in BPA removal efficiency, demonstrating $77-83 \%$ and $73-76 \%$ BPA removal. The large errors in Fig. 5C
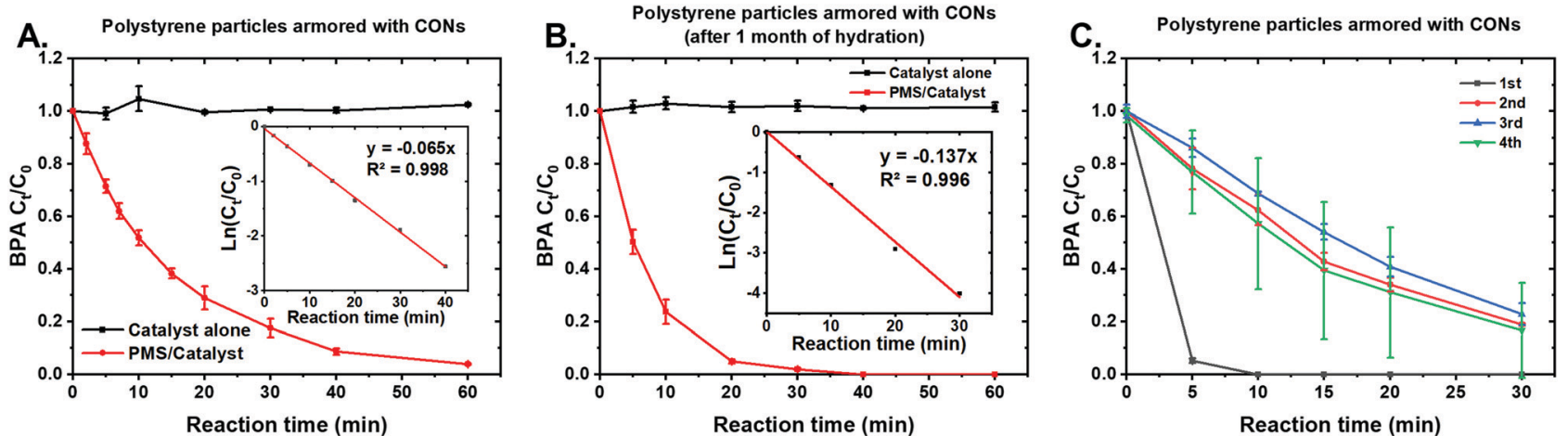

Fig. 5 Catalytic oxidation of BPA by PMS under activation by (A) fresh CON-armored polystyrene particles, (B) CON-armored polystyrene particles after 1 month of hydration, and (C) fresh CON-armored polystyrene particles upon repeated use. Experimental conditions: $[\mathrm{PMS}]_{0}=500 \mu \mathrm{M} ;[\mathrm{BPA}]_{0}=5 \mu \mathrm{M}$; [catalyst] $=0.04 \mathrm{~g} \mathrm{~L}^{-1} ; \mathrm{pH}=5$ with $0.02 \mathrm{M}$ acetate buffer. Error bars stand for data ranges of experimental replicates $(n \geq 2)$. 
and Fig. S14 (ESI $\dagger$ ) might be due to an uneven coating of armored polymer particles onto the two graphite sheets, as this was not observed for the armored particles fully suspended in the solution. The stable performance after the second use indicates that as catalysts $\mathrm{CON}$-armored particles reach stable states and are promising for PMS activation, in contrast to other catalysts whose reactivities continually decrease upon each repeated use.

\section{Conclusions}

Pickering emulsions were prepared by flocculating cobalt oxide nanosheets (CONs) with inorganic salts and agitating in the presence of a hydrophobic monomer, and they were used to prepare CON-armored polymer particles which served as catalysts in the destruction of BPA in water. Optical microscopy images supported that $0.025 \mathrm{M}$ and $0.05 \mathrm{M} \mathrm{NaCl}$ with $1.2 \mathrm{mg} \mathrm{mL} \mathrm{m}^{-1}$ CONs gave the most uniform emulsion droplets which were stable for at least 24 hours. $\mathrm{LiCl}, \mathrm{CsCl}$, and $\mathrm{MgCl}_{2}$ were also examined as flocculants, and of these only LiCl led to formation of stable emulsions. CON-armored polymer particles were prepared by suspension emulsion polymerization and the composition of the composite particles was characterized by TGA, XPS, and SEM, illustrating the presence of both polymer and CONs. The CON-armored particles were used as a catalyst to activate peroxymonosulfate (PMS), an oxidant, in the degradation of bisphenol A (BPA). Full degradation of BPA was observed within $60 \mathrm{~min}$ and the time for complete destruction of BPA was cut in half by aging the particles in a buffer solution. This suggests that the degree of hydration of the nanosheets on the particle surface, which may be related to nanosheet surface area, plays an important role in their catalytic activity. In recyclability studies, the second cycle showed lower BPA removal than the first, but no further decrease in removal efficiency was observed for the third and fourth cycles, unlike other reported catalysts. Practical integration of CON-armored particles for water treatment may require their use as column packing materials or fixing them onto a support, such as activated carbon. The ability to assemble CONs at fluid-fluid interfaces without the use of co-surfactants allows for the creation of new material constructs relevant for environmental remediation, catalysis, and so on, and is expected to be broadly applicable to other architectures templated by oil-water interfaces.

\section{Conflicts of interest}

The authors declare no conflicts of interest.

\section{Acknowledgements}

KP and AS thank AFOSR FA 9550-18-0030 for funding. EP and ME thank NSF DMR CAREER grant \#1955170. KH and HZ thank NSF CHEM grant \#1808406.

\section{References}

1 D. Gonzalez Ortiz, C. Pochat-Bohatier, J. Cambedouzou, M. Bechelany and P. Miele, Current Trends in Pickering Emulsions: Particle Morphology and Applications, Engineering, 2020, 6(4), 468-482.

2 M. Lechuga, M. Fernández-Serrano, E. Jurado, J. Núñez-Olea and F. Ríos, Acute toxicity of anionic and non-ionic surfactants to aquatic organisms, Ecotoxicol. Environ. Saf., 2016, 125, 1-8.

3 M. A. Partearroyo, H. Ostolaza, F. M. Goñi and E. BarberáGuillem, Surfactant-induced cell toxicity and cell lysis: A study using B16 melanoma cells, Biochem. Pharmacol., 1990, 40(6), 1323-1328.

4 B. Brunier, N. Sheibat-Othman, M. Chniguir, Y. Chevalier and E. Bourgeat-Lami, Investigation of Four Different LAPONITE $^{\circledR}$ Clays as Stabilizers in Pickering Emulsion Polymerization, Langmuir, 2016, 32(24), 6046-6057.

5 J. Frelichowska, M.-A. Bolzinger and Y. Chevalier, Pickering emulsions with bare silica, Colloids Surf., A, 2009, 343(1), 70-74.

6 R. Zheng, B. P. Binks and Z. Cui, Pickering Emulsions of Hydrophilic Silica Particles and Symmetrical Organic Electrolytes, Langmuir, 2020, 36(17), 4619-4629.

7 S. M. J. Merritt, A. M. Wemyss, S. Farris, S. Patole, G. Patias, D. M. Haddleton, B. Shollock and C. Wan, Gas Barrier Polymer Nanocomposite Films Prepared by Graphene Oxide Encapsulated Polystyrene Microparticles, ACS Appl. Polym. Mater., 2020, 2(2), 725-731.

8 B. J. Rodier, A. de Leon, C. Hemmingsen and E. Pentzer, Polymerizations in oil-in-oil emulsions using 2D nanoparticle surfactants, Polym. Chem., 2018, 9(13), 1547-1550.

9 Q. Luo and E. Pentzer, Encapsulation of Ionic Liquids for Tailored Applications, ACS Appl. Mater. Interfaces, 2020, 12(5), 5169-5176.

10 M. Ali, T. M. McCoy, I. R. McKinnon, M. Majumder and R. F. Tabor, Synthesis and Characterization of Graphene Oxide-Polystyrene Composite Capsules with Aqueous Cargo via a Water-Oil-Water Multiple Emulsion Templating Route, ACS Appl. Mater. Interfaces, 2017, 9(21), 18187-18198.

11 Q. Luo, Y. Wang, Z. Chen, P. Wei, E. Yoo and E. Pentzer, Pickering Emulsion-Templated Encapsulation of Ionic Liquids for Contaminant Removal, ACS Appl. Mater. Interfaces, 2019, 11(9), 9612-9620.

12 A. de Leon, P. Wei, F. Bordera, D. Wegierak, M. McMillen, D. Yan, C. Hemmingsen, M. C. Kolios, E. B. Pentzer and A. A. Exner, Pickering Bubbles as Dual-Modality Ultrasound and Photoacoustic Contrast Agents, ACS Appl. Mater. Interfaces, 2020, 12(19), 22308-22317.

13 T. D. Gamot, A. R. Bhattacharyya, T. Sridhar, F. Beach, R. F. Tabor and M. Majumder, Synthesis and Stability of Water-in-Oil Emulsion Using Partially Reduced Graphene Oxide as a Tailored Surfactant, Langmuir, 2017, 33(39), 10311-10321.

14 S. C. Thickett and P. B. Zetterlund, Graphene oxide (GO) nanosheets as oil-in-water emulsion stabilizers: Influence of oil phase polarity, J. Colloid Interface Sci., 2015, 442, 67-74. 
15 Q. Luo, Y. Wang, E. Yoo, P. Wei and E. Pentzer, Ionic LiquidContaining Pickering Emulsions Stabilized by Graphene Oxide-Based Surfactants, Langmuir, 2018, 34(34), 10114-10122.

16 M. Yamane, Y. Fadil, M. Tokuda, P. B. Zetterlund and H. Minami, Preparation of Methacrylate Polymer/Reduced Graphene Oxide Nanocomposite Particles Stabilized by Poly(ionic liquid) Block Copolymer via Miniemulsion Polymerization, Macromol. Rapid Commun., 2020, 41(18), 2000141.

17 Y. Fadil, V. Agarwal, F. Jasinski, S. C. Thickett, H. Minami and P. B. Zetterlund, Electrically conductive polymer/rGO nanocomposite films at ambient temperature via miniemulsion polymerization using GO as surfactant, Nanoscale, 2019, 11(14), 6566-6570.

18 H. Cao, M. Escamilla, K. D. Arole, D. Holta, J. L. Lutkenhaus, M. Radovic, M. J. Green and E. B. Pentzer, Flocculation of MXenes and Their Use as 2D Particle Surfactants for Capsule Formation, Langmuir, 2021, 37(8), 2649-2657.

19 K. Edgehouse, M. Escamilla, L. Wang, R. Dent, K. Pachuta, L. Kendall, P. Wei, A. Sehirlioglu and E. Pentzer, Stabilization of oil-in-water emulsions with graphene oxide and cobalt oxide nanosheets and preparation of armored polymer particles, J. Colloid Interface Sci., 2019, 541, 269-278.

20 A. C. de Leon, B. J. Rodier, Q. Luo, C. M. Hemmingsen, P. Wei, K. Abbasi, R. Advincula and E. B. Pentzer, Distinct Chemical and Physical Properties of Janus Nanosheets, ACS Nano, 2017, 11(7), 7485-7493.

21 T. Brugarolas, B. J. Park, M. H. Lee and D. Lee, Generation of Amphiphilic Janus Bubbles and Their Behavior at an AirWater Interface, Adv. Funct. Mater., 2011, 21, 3924-3931.

22 Q. Luo, P. Wei and E. Pentzer, Hollow microcapsules by stitching together of graphene oxide nanosheets with a difunctional small molecule, Carbon, 2016, 106, 125-131.

23 Y. Wang, K. Quevedo and E. Pentzer, Inter-capsule fusion and capsule shell destruction using dynamic covalent polymers, Polym. Chem., 2021, 12(18), 2695-2700.

24 Y. Huang, W. Zhang, G. Ruan, X. Li, Y. Cong, F. Du and J. Li, Reduced Graphene Oxide-Hybridized Polymeric HighInternal Phase Emulsions for Highly Efficient Removal of Polycyclic Aromatic Hydrocarbons from Water Matrix, Langmuir, 2018, 34(12), 3661-3668.

25 L. Yang, C. Jiang, J. Yan, Y. Shen, Y. Chen, L. Xu and H. Zhu, Structuring the reduced graphene oxide/polyHIPE foam for piezoresistive sensing via emulsion-templated polymerization, Composites, Part A, 2020, 134, 105898.

26 R. Bian, R. Lin, G. Wang, G. Lu, W. Zhi, S. Xiang, T. Wang, P. S. Clegg, D. Cai and W. Huang, 3D assembly of $\mathrm{Ti}_{3} \mathrm{C}_{2^{-}}$ MXene directed by water/oil interfaces, Nanoscale, 2018, 10(8), 3621-3625.

27 S. H. Che Man, S. C. Thickett, M. R. Whittaker and P. B. Zetterlund, Synthesis of polystyrene nanoparticles "armoured" with nanodimensional graphene oxide sheets by miniemulsion polymerization, J. Polym. Sci., Part A: Polym. Chem., 2013, 51(1), 47-58.
28 B. J. Rodier, E. P. Mosher, S. T. Burton, R. Matthews and E. Pentzer, Polythioether Particles Armored with Modifiable Graphene Oxide Nanosheets, Macromol. Rapid Commun., 2016, 37(11), 894-899.

29 Y. Fadil, V. Agarwal, F. Jasinski, S. C. Thickett, H. Minami and P. B. Zetterlund, Electrically conductive polymer/rGO nanocomposite films at ambient temperature via miniemulsion polymerization using GO as surfactant, Nanoscale, 2019, 11(14), 6566-6570.

30 Y. Cai, Y. Fadil, F. Jasinski, S. C. Thickett, V. Agarwal and P. B. Zetterlund, Miniemulsion polymerization using graphene oxide as surfactant: In situ grafting of polymers, Carbon, 2019, 149, 445-451.

31 Y. Fadil, S. H. C. Man, F. Jasinski, H. Minami, S. C. Thickett and P. B. Zetterlund, Formation of homogeneous nanocomposite films at ambient temperature via miniemulsion polymerization using graphene oxide as surfactant, J. Polym. Sci., Part A: Polym. Chem., 2017, 55(14), 2289-2297.

32 X. Jin, Y. Duan, D. Liu, X. Feng, W. Li, Z. Zhang and Y. Zhang, CO Oxidation Catalyzed by Two-Dimensional $\mathrm{Co}_{3} \mathrm{O}_{4} / \mathrm{CeO}_{2}$ Nanosheets, ACS Appl. Nano Mater., 2019, 2(9), 5769-5778.

33 D.-L. Nguyen, C.-R. Hsing and C.-M. Wei, Theoretical prediction of superconductivity in monolayer $\mathrm{CoO}_{2}$, Nanoscale, 2019, 11(36), 17052-17057.

34 K. Takada, H. Sakurai, E. Takayama-Muromachi, F. Izumi, R. A. Dilanian and T. Sasaki, Superconductivity in twodimensional $\mathrm{CoO}_{2}$ layers, Nature, 2003, 422(6927), 53-55.

35 H. Volkova, K. Pachuta, K. Crowley, S. K. Radha, E. Pentzer, X. P. A. Gao, W. R. L. Lambrecht, A. Sehirlioglu and M.-H. Berger, Electron microscopy and spectroscopic study of structural changes, electronic properties, and conductivity in annealed $\mathrm{Li}_{x} \mathrm{Co}_{2}$, Phys. Rev. Mater., 2021, 5(1), 015401.

36 H. van Gog, W.-F. Li, C. Fang, R. S. Koster, M. Dijkstra and M. van Huis, Thermal stability and electronic and magnetic properties of atomically thin $2 \mathrm{D}$ transition metal oxides, $n p j$ 2D Mater. Appl., 2019, 3(1), 18.

37 P. Kumbhakar, C. Chowde Gowda, P. L. Mahapatra, M. Mukherjee, K. D. Malviya, M. Chaker, A. Chandra, B. Lahiri, P. M. Ajayan, D. Jariwala, A. Singh and C. S. Tiwary, Emerging 2D metal oxides and their applications, Mater. Today, 2021, 45, 142-168.

38 K. Pachuta, E. Pentzer and A. Sehirlioglu, Evaluating the chemical exfoliation of lithium cobalt oxide using UV-Vis spectroscopy, Nanoscale Adv., 2020, 2(11), 5362-5537.

39 S. K. Radha, K. Crowley, B. A. Holler, X. P. A. Gao, W. R. L. Lambrecht, H. Volkova, M.-H. Berger, E. Pentzer, K. G. Pachuta and A. Sehirlioglu, Ultrathin 2D-oxides: A perspective on fabrication, structure, defect, transport, electron, and phonon properties, J. Appl. Phys., 2021, 129(22), 220903.

40 K. Ferchichi, S. Hbaieb, N. Amdouni, V. Pralong and Y. Chevalier, Pickering emulsion polymerization of polyaniline/ $\mathrm{LiCoO}_{2}$ nanoparticles used as cathode materials for lithium batteries, Ionics, 2014, 20(9), 1301-1314. 
41 C.-H. Lu and P.-Y. Yeh, Ultrafine lithium cobalt oxide powder derived from a water-in-oil emulsion process, J. Mater. Chem., 2000, 10(3), 599-601.

42 N. P. Ashby and B. P. Binks, Pickering emulsions stabilised by LAPONITE ${ }^{\circledR}$ clay particles, Phys. Chem. Chem. Phys., 2000, 2(24), 5640-5646.

43 K. Pachuta, H. Volkova, B. Hirt, M.-H. Berger, E. Pentzer and A. Sehirlioglu, Liquid-Phase exfoliation method to access cobalt oxide nanosheets in pH-neutral solutions, J. Am. Ceram. Soc., 2022, 105, 1904-1912.

44 A. Belfroid, M. van Velzen, B. van der Horst and D. Vethaak, Occurrence of bisphenol A in surface water and uptake in fish: evaluation of field measurements, Chemosphere, 2002, 49(1), 97-103.

45 K. Z. Huang and H. Zhang, Direct Electron-Transfer-Based Peroxymonosulfate Activation by Iron-Doped Manganese Oxide $\left(\delta-\mathrm{MnO}_{2}\right)$ and the Development of Galvanic Oxidation Processes (GOPs), Environ. Sci. Technol., 2019, 53(21), 12610-12620.

46 K. Z. Huang and H. Zhang, Galvanic oxidation processes (GOPs): An effective direct electron transfer approach for organic contaminant oxidation, Sci. Total Environ., 2020, 743, 140828.

47 K. G. Pachuta, E. B. Pentzer and A. Sehirlioglu, Cation deficiency associated with the chemical exfoliation of lithium cobalt oxide, J. Am. Ceram. Soc., 2019, 102(9), 5603-5612.

48 K. Crowley, K. Pachuta, S. K. Radha, H. Volkova, A. Sehirlioglu, E. Pentzer, M.-H. Berger, W. R. L. Lambrecht and X. P. A. Gao, Electrical Characterization and Charge Transport in Chemically Exfoliated 2D $\mathrm{Li}_{x} \mathrm{CoO}_{2}$ Nanoflakes, J. Phys. Chem. C, 2020, 124(38), 20693-20700.

49 T. W. Kim, E. J. Oh, A. Y. Jee, S. T. Lim, D. H. Park, M. Lee, S. H. Hyun, J. H. Choy and S. J. Hwang, Soft-chemical exfoliation route to layered cobalt oxide monolayers and its application for film deposition and nanoparticle synthesis, Chemistry, 2009, 15(41), 10752-10761.

50 J. Huang, S. Zhong, Y. Dai, C.-C. Liu and H. Zhang, Effect of $\mathrm{MnO}_{2}$ Phase Structure on the Oxidative Reactivity toward Bisphenol A Degradation, Environ. Sci. Technol., 2018, 52(19), 11309-11318.

51 L. Hu, G. Zhang, M. Liu, Q. Wang and P. Wang, Optimization of the catalytic activity of $\mathrm{ZnCo}_{2} \mathrm{O}_{4}$ catalyst in peroxymonosulfate activation for bisphenol A removal using response surface methodology, Chemosphere, 2018, 212, 152-161.

52 S. Yang, X. Qiu, P. Jin, M. Dzakpasu, X. C. Wang, Q. Zhang, L. Zhang, L. Yang, D. Ding, W. Wang and K. Wu, MOFtemplated synthesis of $\mathrm{CoFe}_{2} \mathrm{O}_{4}$ nanocrystals and its coupling with peroxymonosulfate for degradation of bisphenol A, Chem. Eng. J., 2018, 353, 329-339.

53 Y. Y. Ahn, E. T. Yun, J. W. Seo, C. Lee, S. H. Kim, J. H. Kim and J. Lee, Activation of Peroxymonosulfate by SurfaceLoaded Noble Metal Nanoparticles for Oxidative Degradation of Organic Compounds, Environ. Sci. Technol., 2016, 50(18), 10187-10197.

54 L. Tang, Y. Liu, J. Wang, G. Zeng, Y. Deng, H. Dong, H. Feng, J. Wang and B. Peng, Enhanced activation process of persulfate by mesoporous carbon for degradation of aqueous organic pollutants: Electron transfer mechanism, Appl. Catal., B, 2018, 231, 1-10.

$55 \mathrm{~J} . \mathrm{Li}, \mathrm{M} . \mathrm{J} . \mathrm{Xu}, \mathrm{G}$. Yao and B. Lai, Enhancement of the degradation of atrazine through $\mathrm{CoFe}_{2} \mathrm{O}_{4}$ activated peroxymonosulfate (PMS) process: Kinetic, degradation intermediates, and toxicity evaluation, Chem. Eng. J, 2018, 348, 1012-1024.

56 H. Zhang and C.-H. Huang, Oxidative Transformation of Fluoroquinolone Antibacterial Agents and Structurally Related Amines by Manganese Oxide, Environ. Sci. Technol., 2005, 39(12), 4474-4483. 